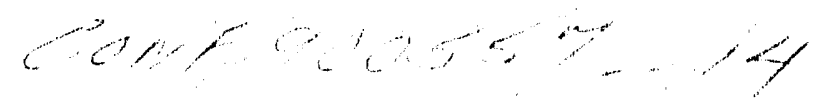

UCRL-JC--103883

DE91 000418

\title{
…...........
}

OCT 051990

\section{Soft $X$-ray Detection with Diamond Photoconductive Detectors}

D.R. Kania, L. Pan, H. Kornblum

P. Bel1, 9.M. Landen, and P. Pianetta
This Pader was Prepared for Submittal to The Eighth Topical Conference on High-Temperature Plasma Diagnostics Hyannis, MA, May 6-10, 1990

May 4, 1990

This is a preprint of a paper intended for publication in a journal or proceedings. Since changes may be made before publication, this preprint is made available with the understanding that it will not be cited or reproduced without the permission of the author. 


\section{Disclaimer}

This document was prepared as an account of work sponsored by an agency of the United States Government. Neither the United States Government nor the University of California nor any of their employees, makes any warranty, express or implied, or assumes any legal liability or responsibility for the accuracy, completeness, or usefulness of any information, apparatus; product, or process disclosed, or represents that its use would not infringe privately owned rights. Reference herein to any specific commerclal products, process, or service by trade name, trademark, manufacturer, or otherwise, does not necessarily constitute or imply its endorsement, recommendation, or favoring by the United States Government or the University of California. The views and opinions of authors expressed herein do not necessarily state or reflect those of the United States Government or the University of California, and shall not be used for advertising or product endorsement purposes. 


\title{
Soft X-ray Detection with Diamond Photoconductive Detectors
}

Thu, May 3, 1990

D. R. Kania, L. Pan ", H. Kornblum, P. Bell, O. N. Landen, and P. Pianetta*

Lawrence Livermore National Laboratory

Livermore, CA 94550

\begin{abstract}
Photoconductive detectors fabricated from natural lla diamonds have been used to measure the $x$-ray power emitted from laser produced plasmas. The detector was operated without any absorbing filters to distort the $x$-ray power measurement. The $5.5 \mathrm{eV}$ bandgap of the detector material practically eliminates its sensitivity to scattered laser radiation thus permitting filterless operation. The detector response time or carrier life time was 90 ps. Excellent agreement was achieved between a diamond PCD and a multichannel photoemissive diode array in the measurement of radiated $x$-ray power and energy.
\end{abstract}




\section{Introduction}

High speed photoconductors (PCDs) have been used to measure the $x$-ray emission from a variety of high temperature plasma sources. $1,2,3,4,5$ using GaAs or $\operatorname{InP}$ as the detector material. In the study of $x$-ray emission from laser plasma interactions the optical sensitivity of these material requires the use of light blocking filters to eliminate scattered laser radiation which distort the flat spectral response of the detector. 3 Diamond has been used as a photoconductive material to eliminate the detector's optical sensitivity and recent measurements have shown that diamond has a flat response between 200 and $2200 \mathrm{eV}^{6}$ Diamond has an extremely small absorption coefficient for energies less than its $5.5 \mathrm{eV}$ bandgap; and, therefore, it is inserisitive to scattered laser radiation with a wavelength greater than $2200 \AA$. These properties make diamond a suitable detector material for

${ }^{1}$ T. F. Deutch, F. Leonberger, A. G. Foyt, and D. Mills, Appl. Phys Lett. 41, 403 (1982).

2L. A. Jones, E. Kallne, L. R. Kania, M. Maestas and R. L. Shepherd, Jour. Appl. Physics 58, 1711 (1986).

${ }^{3}$ D. R. Kania, A. E. Iverson, D. L. Smith, R. S. Wagner, R. B. Hammond, and K. A. Stetlar, Jour. Appl. Phys. 60, 2596(1986).

${ }^{4}$ F. B. Speilman, W. W. Hsing, and D. L. Hanson, Rev. Sci. Instrum. 59,1804 (1988).

${ }^{5}$ C. L. Wang, J. D. Eckels, W. V. Morgan, M. D. Pocha, and D. R. Slaughter, Rev. Sci. Instrum. 57, 2182 (1986).

${ }^{6}$ D. R. Kania, L.S. Pan, P. Bell, O. L. Landen H. Kornblum, P. Pianetta, and M. D. Perry, accepted for publication Jour. Appl. Phys. 
total $x$-ray power measurements from high $Z$ laser produced plasmas. We have used diamond PCDs to measure the $x$-ray yield from uranium disk targets as a function of laser intensity.

\section{Theory of operation}

A single carrier model has been shown to be accurate in modeling diamond PCDs. ${ }^{6}$ In this model the photogenerated carriers, electrons and holes, are treated as a single average carrier. The temporal evolution of the charge carrier density, $n$, is determined by a simple linear rate equation

$$
\frac{\mathrm{dn}}{\mathrm{dt}}=\frac{\mathrm{P}(\mathrm{t})}{\gamma \mathrm{V}}-\frac{\mathrm{n}}{\tau}
$$

where $P$ is the absorbed power, $\tau$ is the carrier lifetime, $V$ is the excited volume, and $\gamma$ is the average energy to form an electron pair. The sensitivity, $S$, in $A / W$ can be derived from this expression

$$
\mathrm{S}=\frac{\mathrm{e} \mu \tau \mathrm{V}_{0}}{\mathrm{~L}^{2} \gamma}=\frac{\mathrm{e} \mu(\boldsymbol{\varepsilon}) \tau \varepsilon}{\mathrm{L} \gamma}
$$

where $V_{0}$ is the applied bias voltage, $\mu$ is the carrier mobility, $\varepsilon$ is the applied electric field, and $L$ is the length of the PCD along $\varepsilon$. Typical sensitivities are approximately $6 \times 10^{-4} \mathrm{~A} / \mathrm{W}$ with carrier lifetimes near 100 ps. 


\section{Experiment}

The detector was a $1 \mathrm{~mm}$ cube of insulating (lla) natural diamond with Ohmic contacts on two opposite faces of the detector. The detector was mounted in a high bandwidth $50 \Omega$ housing which terminated an impedance matched transmission line. The $1000 \mathrm{~V}$ bias voltage was supplied through a capacitive bias tee with a rise time of $60 \mathrm{ps}$. One of the uncontacted faces of the detector faced the radiation source with no filters to distort the measurement or the incident $x$-ray power. A small leakage current, less than $1 \mathrm{nA}$, flows through the detector without $x$-ray illumination. The pulsed $\mathrm{x}$-ray signal was recorded on a high speed oscilloscope.

The detector acts as a radiation controlled resistor in the detection circuit where the decrease in resistance is proportional to the absorbed radiation power. If the output signal is small compared to the applied bias voltage, the signal is proportional to the absorbed power. If the signal level becomes a significant fraction of the applied bias then the output of the PCD must be corrected for the finite impedance of the recording system which is typically $50 \Omega$. This can be corrected for analytically from the expression 


$$
L_{L}=S \Phi=\frac{\text { lout }_{\text {out }}}{1-\text { lout }_{0} \frac{R_{0}}{V_{0}}}
$$

where $\Phi$ is the $x$-ray power incident on the detector, $L_{L}$ is the linear or corrected current, lout is the measured detector current. This correction has been applied to all of the data in this paper.

The radiation source was generated from a laser plasma interaction. One arm of the Nova laser was used to irradiate uranium disk targets. The operating wavelength of the laser was $0.35 \mu \mathrm{m}$. Typically, $2000 \mathrm{~J}$ of laser light in a $1 \mathrm{~ns}$ flat topped pulse was focused upon a $1 \mathrm{~mm}$ diameter, $50 \mathrm{\mu m}$ thick disk of Uranium located at the center of the Nova ten beam target chamber which is four meters from the diamond PCD. The focused laser intensity was varied from $5 \times 10^{13}$ to $3 \times 10^{15} \mathrm{~W} / \mathrm{cm}^{2}$.

The time integrated radiated spectrum and total radiation yield was measured with a photoemissive vacuum diode array (Dante). ${ }^{7} \mathrm{~A}$ low resolution emission spectrum is reconstructed from the output of 13 absolutely calibrated channels covering the energy range rome 180 to $2000 \mathrm{eV}$. The diamond PCD and Dante had nearly identical views of the target, 47 degrees from the target normal. The laser

\footnotetext{
${ }^{7}$ H. N. Kornblum, R. L. Kauffman, and J. A. Smith, Rev. Sci. Instrum. 57, 2179 (1986).
} 
plasma $x$-ray spectrum is dominated by a broad band emission from 200 to $1500 \mathrm{eV}$ with a mean energy of approximately $700 \mathrm{eV}$. A few percent of the radiated power appears in the niobium $L$ lines and the uranium $M$-bands above $2 \mathrm{keV}$. This emission was monitored with an absolutely calibrated $x$-ray crystal spectrograph. A typical composite spectrum is shown in Figure 1. For the $1 \mathrm{~mm}$ cube diamond PCD all of the $x$-rays are stopped in the detector, the incident power equals the absorbed power.

A sequence of 19 uranium laser-plasma experiments were performed to compare the total emitted $x$-ray energy emitted by target as measured with a PCD and the Dante array. The $x$-ray power incident on the PCD varied from 160 to $6700 \mathrm{~W}$ and the linear PCD output current varied from 0.12 to $6.14 \mathrm{~A}$. A plot of the data is shown in Figure 2. An excellent linear relationship exists between the incident $x$-ray power and the linear current output of the PCD. A power law fit yields $I_{L \sim} \Phi^{1.06}$ with $R=0.99$. Using the absolute calibration of the PCD, the conversion efficiency of the uranium disk target as a function of laser intensity was measured. The conversion efficiency is defined as the total radiated $x$-ray energy, assuming an Lambertian radiator, divided by the incident laser energy. This is shown in Figure 3. As expected for the x-ray conversion efficiency for a high $Z$ radiator the conversion efficiency decreases as the laser intensity increases. ${ }^{8}$ A least squares fit

${ }^{8}$ M. D. Rosen, et. al., Phys. Fluids 22, 2020 (1979). 
the the conversion efficiency scales as the incident laser intensity to the -0.26 power.

A few experiments were performed with shaped laser pulses on gold disk targets. For these experiments, the recording system for the Dante detector array was a set of $6 \mathrm{GHz}$ oscilloscopes. The system response time was approximately 100 ps. An identical oscilloscope was attached to a single diamond PCD. The time resolved Dante measurement was analyzed at discrete times to yield the $x$-ray power as a function of time. This requires a complete spectral unfold at each time step and very accurate interchannel timing. The PCD data was linearized according to Equation 2 and compared to the $x$-ray power as measured by the Dante. The results are presented in Figure 4. There is excellent agreement in the measured shaped of the $x$-ray power. This is a further verification of the single carrier model (equation 1), i. e. linear operation of the detector for excitation pulses many time longer than the carrier lifetime. ${ }^{3}$

\section{Conclusion}

The $x$-ray conversion efficiency of uranium has been measured with a single diamond PCD. These measurements are in excellent agreement with an independent measurement using a photoemissive diode array. Also a measurement of the total radiated $x$-ray power made with a diamond PCD is in excellent agreemeni with a time 
resolved measurement using the array. Diamond PCDs promise to be simple and effective detectors for the measurement of $x$-ray power. The device is fast and insensitive to visible radiation and simple to operate.

Acknowledgements

The authors would also like to express their appreciation to $\mathrm{Dr}$. Edward M. Campbell and Dr. Joseph Kilkenny for supporting this work. The efforts of the Nova operations staff were invaluable to the successfiul completion of these experiments. This work was performed under the auspices of the U.S. Department of Energy by Lawrence Livermore National Laboratory under contract No. W-7405Eng-48. 
Figures

1. The emission spectrum from a Uranium laser plasma. The spectrum below $2000 \mathrm{eV}$ was measured with a multichannel filtered vacuum photoemissive diode array and above $2000 \mathrm{eV}$ by a crystal spectrograph.

2. A comparison of the linear current output of a diamond PCD to the $x$-ray power measured with the Dante photoemissive diode array. A near linear relationship is measured. A power law fit to the data scales as $\Phi 1.06$ where $\Phi$ is the $x$-ray power incident on the detector.

3. Uranium x-ray conversion efficiency as a function of laser intensity for $0.35 \mu \mathrm{m}$ light. Conversion efficiency is defined as total radiated $x$-ray energy (assuming a Lambertian source) a divided by the incident laser energy.

4. A comparison of the $x$-ray radiated power as measured by the Dante array with a high speed oscilloscope for each channel and a single PCD recorded on a single high speed oscilloscope. The error bars represent $10 \%$ error expected in the Dante measurement. 

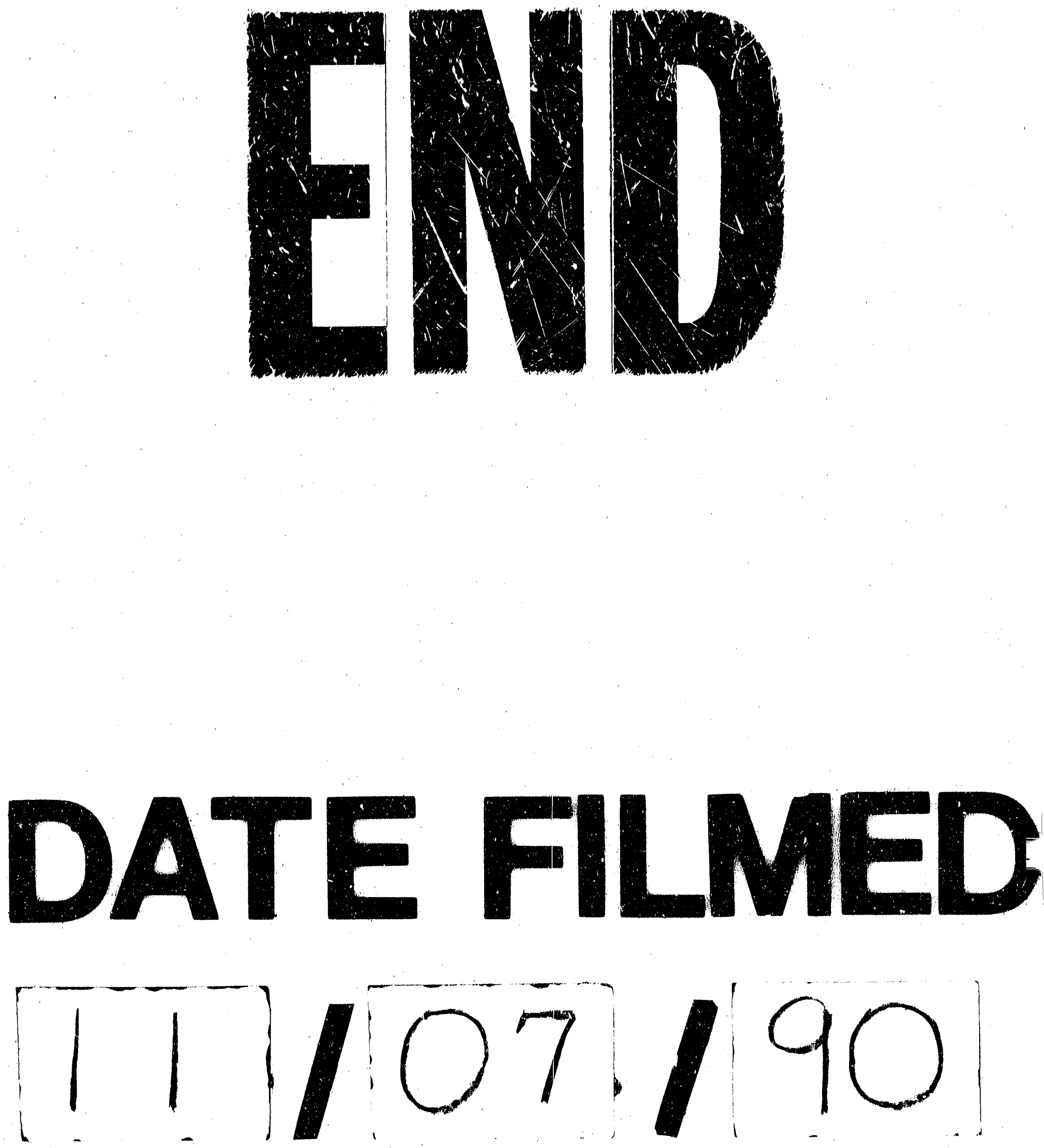